PHYSICAL REVIEW D 94, 099902(E) (2016)

\title{
Erratum: Massive gauge boson pair production at the LHC: A next-to-leading order story [Phys. Rev. D 88, 113005 (2013)]
}

Julien Baglio, Le Duc Ninh, and Marcus M. Weber

(Received 21 October 2016; published 7 November 2016)

DOI: 10.1103/PhysRevD.94.099902

The combination of the theoretical uncertainties of $W^{+} Z$ and $W^{-} Z$ channels into a single $W Z$ channel was done incorrectly [pages 21-22, point (iii)]. Instead of summing the absolute errors in pb we summed the percentage errors, leading to an overestimation of the theoretical uncertainties on the combined $W Z$ channel.

The correct expressions should read $\sigma_{W Z}^{\text {th }}=18.3_{-1.0}^{+1.2} \mathrm{pb}$ at $7 \mathrm{TeV}$ and $\sigma_{W Z}^{\text {th }}=22.7_{-1.1}^{+1.4} \mathrm{pb}$ at $8 \mathrm{TeV}$. The agreement with ATLAS and CMS results quoted in the point (iii) should read as follows: at $7 \mathrm{TeV}$ a $0.3 \sigma$ agreement with ATLAS and a $0.4 \sigma$ agreement with CMS, at $8 \mathrm{TeV}$ a $1.2 \sigma$ agreement with ATLAS. Figure 23 should be corrected as well, and the following Fig. 1 replaces the corresponding $W Z$ plot in Fig. 23 of the paper.

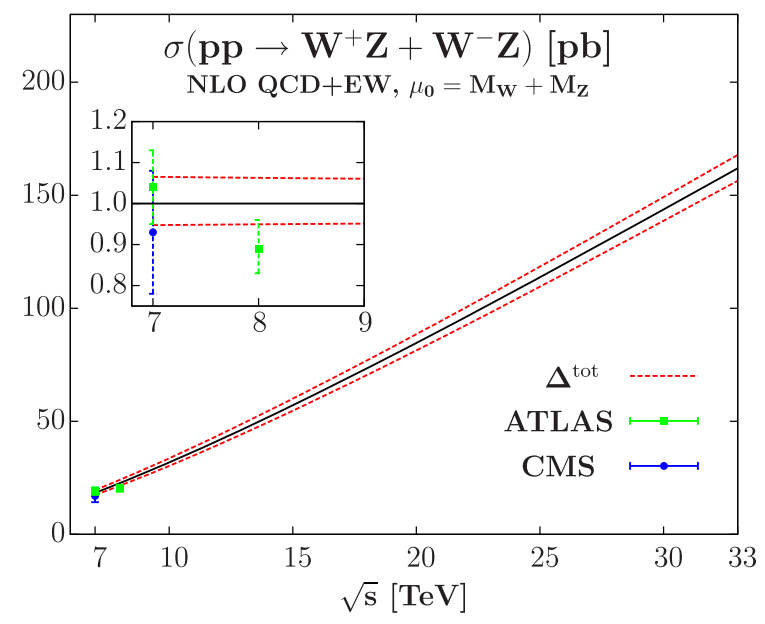

FIG. 1. The NLO QCD + EW total cross section (black/full, in pb) of the process $p p \rightarrow W^{+} Z+W^{-} Z$ at the LHC as a function of the center-of-mass energy $\sqrt{s}$ (in $\mathrm{TeV}$ ) including the total theoretical uncertainty (red/dashed) as discussed in the text. The inset shows the relative deviation from the central cross section, and the experimental data points are also displayed on the main figure. 